\title{
SCIENZA, FENOMENOLOGIA E RIDUZIONE
}

\author{
CIÊNCIA, FENOMENOLOGIA E REDUÇÃO \\ SCIENCE, PHENOMENOLOGY AND REDUCTION
}

Paul Gilbert*

\section{SOMMARIO}

Husserl si chiedeva se la filosofia potesse essere una "scienza rigorosa". Questa domanda avrà ancora un interesse? Non dovremmo però contestare l'unilateralità della deriva culturale dei nostri tempi e rivendicare per la riflessione fondamentale nuovi spazi d'interrogazione? Le scienze sono credibili soltanto perché offrono la possibilità di alimentare la potenza della tecnica? Non dobbiamo porre invece la domanda sul loro fondamento razionale; criticare la mentalità che si accontenta del loro successo tecnico? Tenteremo di rispondere a queste domande leggendo alcuni testi di Edmund Husserl, Martin Heidegger e Michel Henry. Il nostro intento è di capire il significato del termine "riduzione" in fenomenologia.

PAROLE CHIAVE: Husserl. Heidegger. Michel Henry. Fenomenologia. Riduzione.

\section{RESUMO}

Husserl questionou se a filosofia pode ser uma "ciência rigorosa". Esta questão ainda terá algum interesse? Não deveremos então desafiar a unilateralidade do movimento cultural dos nossos tempos e reivindicar novos espaços de interrogação destinados à reflexão fundamental? As ciências são críveis só porque oferecem a possibilidade de alimentar a potência da técnica? Mas não seria o caso de colocarmos em questionamento seus fundamentos racionais e criticar a mentalidade que se contenta com seu sucesso técnico? Tentaremos responder a estas perguntas, lendo alguns textos de Edmund Husserl, Martin Heidegger e Michel Henry. O nosso objetivo é compreender o significado do termo "redução" no âmbito da fenomenologia.

PALAVRAS-CHAVE: Husserl. Heidegger. Michel Henry. Fenomenologia. Redução.

\footnotetext{
*Ex-catedrático de metafísica da Pontificia Università Gregoriana de Roma. E-mail: Gilbert@ unigre.it.
} 


\section{ABSTRACT}

Husserl questioned whether philosophy can be a "rigorous science." This question will still have some interest? We should not, however, challenge the one-sidedness of the cultural movement of our times and claim new interrogation spaces for critical reflection? Science is credible only because they offer the possibility of feeding the power of the technique? But we should not ask about their rational grounds and criticize the mentality that is content with its technical success? We will try to answer these questions, reading some texts of Edmund Husserl, Martin Heidegger and Michel Henry. Our goal is to understand the meaning of the term "reduction" in phenomenology.

KEYWORDS: Husserl. Heidegger. Michel Henry. Phenomenology. Reduction.

\section{INTRODUZIONE}

La cultura odierna non sa come orientare la nostra vita. Normalmente, una cultura serve prima di tutto a questo. Oggi siamo lasciati soli. Fino a poco tempo fa, dire che un uomo era colto significava che aveva molte competenze - anche se elementari - in scienza e letteratura, che lo animava un carattere intrepido, che s'interessava di tutto, che sapeva anche ascoltare molte opinioni senza illudersi. Per un atteggiamento spontaneo, amava tutto ciò che riguardava l'umano, con profondità ma anche insoddisfazione spontanea. Un uomo colto era paradossale perché, da una parte, voleva conoscere meglio la complessità umana e, dall'altra, sapeva che non poteva mai trovare qualche soluzione completa e definitiva a qualsiasi serio problema umano. Sembra che la cultura odierna non cammini più così. È divenuta tanto frammentata quanto dogmatica, sicura di sé nel poco che conosce. Ha paura delle avventure intellettuali. Un uomo colto s'interessava di tutto con un certo distacco, un certo scetticismo, spesso con una raffinata ironia. Gli uomini contemporanei non sono più colti in questo modo. Sono super specializzati, ignoranti di tante cose e, informati in tempo "reale", presumono di sapere tutto.

La cultura filosofica e scientifica accede ai media solo nelle ore più buie della notte. Sarà quindi da abbandonare ai nottambuli? Husserl si chiedeva se la filosofia potesse essere una "scienza rigorosa". Questa domanda avrà ancora un interesse? Non dovremmo però contestare l'unilateralità della deriva culturale dei nostri tempi e rivendicare per la riflessione

Sapere aude - Belo Horizonte, v. 7 - n. 12, p. 301-329, Jan./Jun. 2016 - ISSN: 2177-6342 
fondamentale nuovi spazi d'interrogazione? Le scienze sono credibili soltanto perché offrono la possibilità di alimentare la potenza della tecnica? Non dobbiamo porre invece la domanda sul loro fondamento razionale; criticare la mentalità che si accontenta del loro successo tecnico? Tenteremo di rispondere a queste domande leggendo alcuni testi di Edmund Husserl, Martin Heidegger e Michel Henry ${ }^{1}$.

Il nostro intento è di capire il significato del termine "riduzione" in fenomenologia. Questo termine ha conosciuto alcune avventure. Indica, infatti, per un fenomenologo il metodo più radicale per fondare il senso delle attività umane, comprese quelle scientifiche. Notiamo però, prima di cominciare, che il termine "scienza" è ambiguo. Traduce, infatti, due termini greci, theōria e epistēmē. La parola theōria indica un atteggiamento "oggettivo", che considera prima di tutto ciò che si desidera conoscere, senza intervenire su di esso. La mente è qui rispettosa di ciò che è e che lascia venire in presenza: ciò che si vuole conoscere è riconosciuto, dato per primo, e la theōria rispetta questa priorità. Il termine epistēme, invece, significa l'attività interpretativa di ciò che si sta guardando, costituisce dunque un "saper fare", un lavoro prudente che, da un lato, accoglie l'oggetto conosciuto adattandolo alle nostre capacità di comprensione e, dall'altro lato, critica le nostre possibilità conoscitive perché siano più competenti per realizzare una corretta accoglienza delle cose.

Il termine theōria suggerisce quindi che ci sia un dominio che trascende la ragione umana, anzi che sia per principio inaccessibile alle prese della ragione; una cosa non può mai essere conosciuta "in sé", o nelle sue proprietà più essenziali, in un modo totalmente esatto e completo. Non è senza motivo che, nel platonismo, la theōria si situa al vertice delle nostre possibilità di conoscenza, oltre i limiti del discorso, benché questi limiti non condannino al silenzio. Ci sono, infatti, procedimenti della ragione che non terminano mai, benché non siano inutili. Per esempio, la dialettica ascendente che Platone spiega all'inizio del quinto libro della sua Repubblica (mi riferisco alla celeberrima metafora della caverna) non termina nel vedere distintamente le idee, ma nell'essere abbagliati da esse. La theōria non potrà mai pretendere di essere esatta; sarà sempre giudicata approssimativa, perché senza corrispondenza interamente determinabile con la sensibilità e le nostre competenze in argomentazioni logiche.

\footnotetext{
${ }^{1}$ Indicheremo nel testo stesso con abbreviazioni le pagine delle principali opere citate:

(C): E. HUSSERL. La crisi delle scienze europee e la fenomenologia trascendentale;

(D): E. HuSSERL; M. HEIDEGGER. Fenomenologia. Storia di un dissidio (1927);

(F): E. HUSSERL. La filosofia come scienza rigorosa;

(G): E. HUSSERL. Glosse a Heidegger;

(Q): M. HENRY. «Quatre principes de la phénoménologie».
} 
Segue da queste considerazioni che il sintagma "scienza teorica" sia problematico per essenza. La parola epistēmē, invece, ha un significato più facilmente comprensibile, perché rimanda alla pratica delle scienze, alla visibilità delle loro operazioni e costruzioni, alla pratica delle loro metodologie, senza che si possa dedurre da ciò che termineranno in un sapere definitivamente stabilito. La "riduzione" fenomenologica ha qualcosa a che vedere con l'epistèmē.

\section{Edmund Husserl e le scienze}

La critica di Husserl alla "scienza" è costante, dai Prolegomeni alla logica pura del $1900^{2}$, il volume che introduce alle Ricerche logiche, fino alla Crisi delle scienze europee, del 1935. Per "scienza", dobbiamo intendere anzitutto le scienze empiriche, fisiche o umane che siano, psicologiche soprattutto, meno le scienze matematiche come la geometria cui Husserl consacrò il suo ultimo lavoro, L'origine della geometria, terminato nel 1936 ma pubblicato solo nel 1954. Il punto messo in discussione da Husserl è il metodo delle scienze empiriche, il senso della loro pratica, dei loro modelli d'investigazione, e particolarmente dei modelli che la psicologia scientifica adotta e intende seguire per esibire la sua scientificità.

Come primo testo che introdurrà al pensiero di Husserl sulla scienza, scelgo un lungo articolo pubblicato nel 1911, La filosofia come scienza rigorosa, e in particolare le sue prime pagine (le parti che seguono trattano la prima del naturalismo e la seconda dello storicismo). Siamo pochi decenni dopo la pubblicazione della Introduzione alle scienze dello spirito (1883) in cui Wilhelm Dilthey distingueva le scienze naturali e, appunto, le scienze dello spirito. Ora questa distinzione non è stata pensata radicalmente da Dilthey stesso; era di semplicità (natura - l'oggettività) e di complessità (spirito - la riflessività) più che di metodo. Sappiamo, però, che la scienza è prima di tutto un metodo e che le norme della causalità vi s'impongono sempre. Dilthey aveva distinto la spiegazione (in scienze naturali) e la comprensione (in scienze dello spirito). "Comprendere" è essenziale in scienze umane, che richiedono una certa empatia con il conosciuto. L'empatia dovrebbe quindi essere una modalità della scientificità. È però poco probabile che lo sia. La storia, di cui Dilthey si occupava prevalentemente, era comunque una scienza di eventi, cioè di fatti. I fatti umani

${ }^{2}$ E. HUSSERL, Prolegomeni a una logica pura, p. 17-197.

Sapere aude - Belo Horizonte, v. 7 - n. 12, p. 301-329, Jan./Jun. 2016 - ISSN: 2177-6342 
possono, però, essere intesi come fatti della natura e trattati con la causalità che vale per le scienze empiriche? Nel suo articolo, Husserl rimanda a una pubblicazione molto recente di Dilthey sulla diversità delle Weltanschauugen, cioè delle visioni del mondo ${ }^{3}$. Le visioni del mondo, secondo Dilthey, non permettono di costruire un sapere scientifico dei fatti umani. Manca loro la sicurezza che la metodologia dà alle scienze della natura, in particolare un metodo fedele alle relazioni causali. Senza esitazioni, però, Husserl critica questa tesi. Da un lato, si può rimandare alla storia della stessa fisica, ai cambiamenti dei suoi paradigmi che provano quanto la scienza non abbia la sicurezza che Dilthey le attribuisce. Dall'altro lato, è necessario che l'empatia si sottometta al metodo empirico? Per Dilthey, «la storia [è] la scienza empirica dello spirito in generale» (F 76). Husserl non può non contestare una simile affermazione. Nella pratica della psicologia che aveva conosciuto durante la sua formazione a Vienna, gli schemi metodologici erano quelli della causalità nelle scienze naturali, del "naturalismo". Questa presupposizione metodologica deveva essere combattuta.

La prima parte dell'articolo lo sottolinea con vigore, come lo facevano già i Prolegomeni undici anni prima. La scientificità della psicologia è da interrogare in riferimento all'originalità delle scienze dello spirito diversamente da Dilthey. I fatti della storia non sono bene intesi, se vengono utilizzate problematiche naturalistiche, costruite per interpretare i fatti mediante altri fatti. Lo storicismo di Dilthey non poteva fare a meno del naturalismo. La prima cosa da fare, quindi, era di smascherare la pretesa naturalista e di interrogare il vero significato dell'idea di rigore, nella scienza in generale. Le caratteristiche di una scienza rigorosa sono dispiegate da Husserl nelle prime righe del suo articolo del 1911: una scienza rigorosa è «in grado di soddisfare le più elevate esigenze teoretiche e di rendere possibile [...] una vita regolata da pure norme razionali» (F 3). I due domini della teoria e della pratica sono così collocati sotto l'autorità della ragione; si uniscono a tal punto che la teoria possa imporsi alla pratica, e la pratica alla teoria ${ }^{5}$. Ogni teoria o lavoro intellettuale implica un aspetto

\footnotetext{
${ }^{3}$ Vedi W. DILTHEY, «I tipi di visione del mondo e la loro formazione nei sistemi metafisici», p. 581-635: la vita «si è oggettivata nelle sue manifestazioni, da parte della comprensione e dell'interpretazione, più compiutamente che in ogni consapevolezza e in ogni apprendimento del proprio Erlebnis» (p. 587). L'originale è del 1911.

${ }^{4}$ Per Husserl come per tutta la tradizione speculativa, le matematiche sono a priori, le scienze empiriche sono invece a posteriori. A Vienna, si cercava di spiegare i procedimenti matematici alla luce di una psicologia naturalista che non poteva non terminare in qualche relativismo o impossibilità d'affermazioni certissime; è ovvio che 2 e 2 fanno 4, indipendentemente dello stato psicologico di chi lo dice. Questa questione era stata discussa già nei Prolegomeni del 1900, cui l'articolo del 1911 rimanda (F 75, n. 38). Il problema è quello della relazione tra l'a priori e l'a posteriori.

${ }^{5}$ Per "pratica", si possono intendere le situazioni mentali che fanno vedere o meno alcune realtà; in questo senso, le pratiche influiscono, infatti, sulle costruzioni teoretiche. Per esempio, «le capacità per la pura teoria
} 
pratico, la sua epistēme, il suo modo di procedere. Ora tutte le scienze si sviluppano sotto alcune idee o strutture fondamentali. Il lavoro del filosofo riguardo a una scienza dello spirito consiste quindi nell'evidenziare le sue strutture fondamentali.

La pratica scientifica non è soltanto teoretica. L'epistemologo Husserl ha cura di non eliminare dalla sua prospettiva il saper-fare della scienza. Una comprensione solamente teoretica della scienza sarebbe troppo ingenua. Distrarrebbe prima di tutto dalla storia delle scienze, dalle loro evoluzioni e approfondimenti. La scienza è sempre alla ricerca, non termina mai. Tuttavia, l'ideale di rigore scientifico, e la speranza di acquistare una conoscenza certa, cioè solida e definitiva, non sono illusori; sostengono, infatti, tutto il progresso scientifico. Un risultato scientifico provvisorio non significa un risultato arbitrariamente relativo e senza un senso affidabile. D'altronde, la filosofia partecipa della stessa situazione della scienza. «In nessuna epoca del suo sviluppo la filosofia è stata in grado di soddisfare la pretesa di essere scienza rigorosa» (F 3), benché gli autori l'abbiano sempre desiderato. Per esempio, la filosofia post-rinascimentale va, come la classica, «in una direzione essenzialmente unitaria» (F 3), essendo l'unità il fine della coerenza razionale; Cartesio aveva proposto la stessa prospettiva ${ }^{6}$, però senza offrire un sistema deduttivo che potesse convincere universalmente. La filosofia ha gli stessi “oggetti” della fisica e della storia, ma non convince né gli scienziati né gli stessi filosofi che potesse ottenere un risultato definitivamente certo, «il che mostra come il senso autentico dei problemi filosofici non sia mai stato scientificamente chiarito» (F 4). Ci saranno mai, però, chiarificazioni scientifiche soddisfacenti? La storia delle scienze parteggia una risposta negativa. Che cosa significa, dunque, chiarire scientificamente il senso dei problemi filosofici?

Una vera scienza, diceva la tradizione antica, s'insegna, e perciò la sua struttura è prettamente matematica. Per Tommaso d'Aquino, per esempio, la ratio è legata alle esperienze del mondo fisico in cui si constatano continui cambiamenti che provengono dalle modifiche delle relazioni cosali; l'intellectus è invece la funzione conoscitiva dei principi

minacciavano di venire meno $[\ldots]$, le forze religiose soffocavano la libertà della ricerca teoretica» (F 3). La "pratica" può però essere il cammino metodologico di qualsiasi scienza; l'idea husserliana di "coscienza costituente" potrebbe essere di questo genere, come lo sanno le scienze contemporanee.

${ }^{6}$ Vedi il celebre passo in cui Cartesio illustra l'organizzazione scientifica del sapere umana con la metafora dell'albero: DESCARTES. «Lettera dell'Autore a colui che ha tradotto il libro» (i Principi della filosofia), in ID., Opere, 1637-1649, p. 2230.

Sapere aude - Belo Horizonte, v. 7 - n. 12, p. 301-329, Jan./Jun. 2016 - ISSN: 2177-6342 
primi e stabili; quanto alla matematica, essa è l'unica scienza che s'insegna disciplinabiliter ${ }^{7}$, cioè con metodi sicuri perché logici e formali; a essa non corrisponde poi alcuna facoltà conoscitiva paragonabile alla ratio e all'intellectus. Per Husserl, alla filosofia «mancano ancora problemi, metodi e teorie concettualmente ben definiti e pienamente chiariti nel loro senso» (F 5); la preoccupazione è qui della "disciplina", non delle facoltà e dei loro "oggetti". Kant aveva avvertito similmente che non s'insegna la filosofia, ma a filosofare ${ }^{8}$; il filosofo non insegna cose, ma accompagna i suoi ascoltatori nei modi di pensare, di condurre le proprie riflessioni. Per Husserl, «nessuna persona ragionevole metterà in dubbio la verità oggettiva o la probabilità oggettivamente fondata delle stupefacenti teorie della matematica e delle scienze naturali» $\left(\mathrm{F}^{9}\right)$. Il filosofo non gode però di un simile sapere "oggettivo"; anzi mette eroicamente in dubbio ogni sapere che pretende una simile "oggettività". S'interessa piuttosto all'atto di conoscere e alle sue condizioni trascendentali, e cerca le chiavi di una filosofia rigorosa, a questo livello non filosofico e più che logico.

I filosofi hanno sempre sognato qualche sistema dottrinale completo e perfetto nei particolari, ma non l'hanno mai realizzato. Non c'è nemmeno qualche scientificità filosofica che sia universalmente accettata. Dovremmo quindi abbandonare l'idea che la filosofia possa essere «una scienza rigorosa» (F 8)? È inutile sperare qualche nuovo sistema che accantonerà quelli che sono stati presentati nei tempi passati. È ugualmente inutile prendere elementi dalle filosofie, sparsi nella storia, per comporre con essi una costruzione progressiva, «pietra dopo pietra» (F 8), suscettibile di completare un sistema onnicomprensivo. L'unica possibilità è quella di andare alle fonti, di "rifondare" la ricerca filosofica, come d'altronde l'avevano già tentato Platone, i moderni, Kant e Fichte, anche se nessuno abbia preteso di mettere a posto un “sistema" filosofico chiuso. «Solo nella filosofia romantica si assiste ad un mutamento» (F 9), nota Husserl, infatti con Hegel. Come però afferma Husserl con ironia, in Hegel manca «quella critica della ragione che prima fra tutte rende possibile la scientificità filosofica» $(\mathrm{F}$ 9). A causa di questa debolezza, proprio la filosofia hegeliana ha generato indebolimenti e falsificazioni «dell'impulso alla costituzione della rigorosa scienza filosofica» (F 9); ha fatto

\footnotetext{
${ }^{7}$ Tommaso D’Aquino, Commenti a Boezio, q. 6, a. 1, p. 325.

${ }^{8}$ Vedi I. KANT, «Annuncio del programma delle sue lezioni per il semestre d'inverno 1765-1766» (Ak. II, p. 306).

9 Il termine "oggettivamente" vale qui insieme per la matematica e le scienze naturali, con significati però diversi. Corrisponde quindi a una intenzione globalmente unica della mente, ma con capacità differenti, tutte determinate dalla necessità, ma l'una a priori (secondo le norme della sola ragione) e l'altra a posteriori (cioè a partire dalle osservazioni empiriche).
} 
nascere il naturalismo e lo scetticismo che sono alla radice della filosofia contemporanea e della moda delle "visioni del mondo" (Weltanschauung).

Husserl conclude che è necessario animare la filosofia con «l'intenzione di una rifondazione $[\ldots]$ nel senso di una scienza rigorosa» (F 11). Che cosa sarebbe però una "scienza rigorosa"? Il naturalismo, quale teoria filosofica, aveva tentato questo sforzo, ma «in una forma che è fondamentalmente errata da un punto di vista teoretico e che costituisce da un punto di vista pratico un crescente pericolo per la nostra cultura» (F 11). Come fare, quindi? Husserl non lo dice nel suo scritto del 1911, verosimilmente (l'esperienza è comune) perché vedere un problema non significa evidenziare gli elementi di una soluzione soddisfacente. Una successiva riflessione è indispensabile. L'articolo del 1911 si ferma quindi alla decostruzione del naturalismo e dello storicismo. Mette però in scena gli elementi di soluzione, per esempio un senso della storia che integra gli atti della coscienza su cui Husserl aveva già riflettuto nel suo corso del 1904-1905 sulla Coscienza interna del tempo, che non riusciva però a pubblicare in una forma che lo soddisfacesse ${ }^{10}$. Abbiamo qui le basi fenomenologiche della "riduzione", del ritorno della coscienza sui suoi atti. Ogni scienza s'inserisce in una prospettiva della mente umana di cui la fenomenologia evidenzia la struttura originale a priori, trascendentale, prima di ogni oggettivizzazione del conosciuto o di ogni pretesa che esso sia "reale" 11

La Crisi, l'ultimo grande testo di Husserl, riprende la riflessione sulla tendenza naturalistica delle scienze contemporanee, compresa la psicologia. Ci soffermeremo adesso sulla prima parte del libro, intitolata «La crisi delle scienze quale espressione della crisi radicale di vita dell'umanità europea» (C 33). Si riconosce prima di tutto «il rigore scientifico di tutte queste discipline, l'evidenza delle loro operazioni teoretiche e dei loro successi» $(\mathrm{C}$ 34). Si conferma poi «il contrasto tra la "scientificità" di questo gruppo di scienze e la "nonscientificità" della filosofia» (C 34). Detto questo, non possiamo non riconoscere la perdita «per la vita» (C 34, titolo del § 2) del significato delle scienze così spiegate. La critica del naturalismo vale anche per la psicologia e la sua pretestuosa scientificità, che scarta ogni preoccupazione per il senso della vita. «Le mere scienze di fatti creano meri uomini di fatto» (C 35) dice Husserl lapidariamente, e perciò esse escludono dalle loro prospettive le domande

\footnotetext{
${ }^{10}$ Edith Stein, allora assistente di Husserl, ci provò nel 1916.

${ }^{11}$ Per un approfondimento della "riduzione" in Husserl, vedi St. BANCALARI, Logica dell'epochè. Per una introduzione alla fenomenologia della religione, p. 135-155.
}

Sapere aude - Belo Horizonte, v. 7 - n. 12, p. 301-329, Jan./Jun. 2016 - ISSN: 2177-6342 
su «noi uomini in quanto soggetti di [...] libertà» (C 36). Nelle scienze storiche, scienze $a$ priori umane come la psicologia, il sapere accademico esige pure «che lo studioso eviti accuratamente qualsiasi presa di posizione valutativa, tutti i problemi concernenti la ragione o la non-ragione dell'umanità tematizzata e delle sue formazioni culturali» (C 36). Assistiamo però così a un allontanamento delle scienze dell'uomo da una «un'umanità autentica» (C 35).

Nell'antichità, i pensatori si preoccupavano della «forma "filosofica" dell'esistenza» (C 37). Era chiara per loro la «capacità di dare liberamente [...] a tutta la propria vita, regole fondate sulla pura ragione» (C 37). Il rinascimento ha ripreso l'essenziale di questa esigenza umana, assumendo i secoli nominalisti immediatamente precedenti. «All'autonomia teoretica succede quella pratica. Nell'ideale del Rinascimento, l'uomo antico è quello che plasma se stesso esclusivamente in base alla libera ragione» (C 37). Il concetto contemporaneo di scienza naturalistica, quindi, non è né antico né originario, è solo un «concetto residuo» (C 38) di cui si è tolto l'importanza dell'attenzione agli aspetti propriamente umanistici del sapere.

Diviene quindi urgente ispirarsi agli Antichi e al Rinascimento, e preoccuparci dell'«ordine di senso dell'essere e quindi dei problemi dell'essere» (C 39). Husserl evoca in questo momento l'«Inno alla gioia» di Schiller, che Beethoven utilizzò nell'ultimo movimento della sua nona sinfonia, l'inno dell'Europa che la Commissione europea ha definitivamente voluto senza parole nel 2004 per evitare ogni difficoltà di traduzione da una lingua europea a un'altra, e per eliminare ogni allusione a Dio, origine dell'unione di tutte le società umane ${ }^{12}$. La rinuncia a ogni sistema scientifico è stata così confermata dai responsabili europei. Ci sono stati però alcuni filosofi, attorno all'anno 35, che hanno desiderato comprendere i «veri motivi di un secolare fallimento» (C 40) e che lottano contro l'affondamento del progetto filosofico nel positivismo ambiente, «mentre i più trovarono e continuano a trovare con molta disinvoltura le formule capaci di tranquillizzare se stessi e i propri lettori» (C 40).

La filosofia manifesta così che è un problema per se stessa. «Il crollo della fede in una filosofia universale capace di guidare l'uomo nuovo ${ }^{13}$, indica appunto il crollo della fede nella "ragione" [...] intesa nel senso in cui gli antichi contrapponevano l'epistēmē alla doxa» (C 42). C'è però una differenza tra una necessità cercata senza mai raggiungerla come si vorrebbe, e una necessità cui si rinuncia, lasciandola crollare definitivamente. Crolla insieme

\footnotetext{
${ }^{12}$ L'inno comincia, infatti, con queste parole: «Freude, schöner Götterfunken, Tochter aus Elysium!».

${ }^{13}$ Non possiamo non indovinare qui un'allusione ironica al nazismo da poco al potere in Germania.
} 
«la fede nel senso della storia, nel senso dell'umanità, nella sua libertà in quanto attiva possibilità dell'uomo di conferire un senso razionale alla sua esistenza umana individuale e umana in generale» (C 42). L'obbiettivo dei filosofi era «il chiarimento dei dati ultimi e dei fini e dei mezzi che essi suggeriscono in un modo definitivamente e veramente razionale» $(\mathrm{C}$ 45). Questo progetto rimane. Come? «Attraverso una considerazione critica di ciò che nella propria finalità e nel proprio metodo rivela quell'aderenza ultima e autentica alla propria origine che, una volta penetrata, lega a sé apoditticamente la volontà» (C 47). Le scienze contemporanee si accontentano di spiegare i fatti con le cause antecedenti. Husserl insiste, invece, sugli aspetti umani del sapere, che hanno una sfumatura teleologica.

La Crisi, che è stata scritta dal 1934 al 1937, non sarà pubblicata da Husserl stesso, autore odiato dai nazisti. Una bozza è stata però proposta in una conferenza del 7 maggio $1935^{14}$. Vi si tratta dell'umanità europea, della sua tradizione filosofica e umanistica, che è stata piegata dal razionalismo nell'empirismo degli ultimi secoli, ciò che ha provocato la "crisi europea" sotto gli occhi di tutti in Germania, in Spagna, in Francia, ecc. Secondo Husserl, la crisi chiedeva una risposta precisa: «l'idea storico-filosofica (il senso teleologico) dell'umanità europea» (C 328) doveva essere assunta di nuovo. Non si nega il significato scientifico del razionalismo, alla condizione però che esso non decada in "naturalismo" o "oggettivismo" e che rinasca il dinamismo teleologico che non si accontenta di produrre oggetti tecnicamente disponibili.

Per penetrare il groviglio della "crisi", attuale, era indispensabile elaborare il concetto Europa in quanto teleologia storica di fini razionali infiniti; era indispensabile mostrare come il mondo europeo sia nato da idee razionali, cioè dallo spirito della filosofia (C 358).

Husserl è veramente un autore a noi contemporaneo.

\section{Martin Heidegger e la fenomenologia husserliana}

Nel 1938, Heidegger propose una conferenza sull'«Epoca dell'immagine del mondo» ${ }^{15}$ : il destino delle scienze contemporanee consisterebbe nel costruire immagini e rappresentazioni del mondo (Weltbildes), non nel conoscere la realtà in sé stessa. Siamo pochi

\footnotetext{
${ }^{14}$ «La crisi dell'umanità europea», in C 328-358.

${ }^{15}$ M. HeIDEGGER, L'epoca dell'immagine del mondo, p. 71-101.
}

Sapere aude - Belo Horizonte, v. 7 - n. 12, p. 301-329, Jan./Jun. 2016 - ISSN: 2177-6342 
anni dopo la conferenza di Husserl sulla Crisi, una riflessione che ha probabilmente influenzato Heidegger, il suo successore a Friburgo, ma con sfumature di notevole interesse. L'uno e l'altro affrontarono un problema che s'imponeva in Germania in preparazione della guerra, cioè il processi di riarmo cui partecipavano le fabbriche e i laboratori più importanti dell'industria tedesca. Per Husserl, il correspettivo della produzione tecnica fu l'occultamento dello spirito europeo: la scienza tecnicizzata può fare a meno del mondo della vita, del Lebenswelt e della sua finalità infinita. Heidegger, che era stato poco prima Rettore di turno dell’Università di Friburgo, considerava anche la scienza in relazione alla tecnica, figlia del naturalismo oggettivistico. Il suo tristamente celebre discorso d'insediamento al rettorato, nel 1933, insisteva sulla gerarchia delle scienze insegnate nell'università e sulla precedenza assoluta della riflessione filosofica, una gerarchia che la potenza militare stava però per capovolgere completamente. L'ambiguità della scienza era ovvia. La teleologia umanistica su cui Husserl insisteva nel 1935 si trasformava sempre di più in progetti tecnici realizzati scientificamente. Ciò, nella conferenza del 1938, era oggetto della critica di Heidegger perché le strutture economiche sostenevano quei progetti per rispondere ai propri interessi e senza considerazione per l'essenza dell'Esserci ${ }^{16}$. Le idee d'intenzionalità e di Lebenswelt venivano così completamente pervertite.

La riflessione di Heidegger riguardo alla scienza insisté sulla sua dipendenza dalla tecnica, anche con approfondimenti originali, per esempio quando, nella conferenza del 1951 «La questione della tecnica» ${ }^{17}$, l'analisi fenomenologica evidenziava l'appartenenza delle scienze alla struttura della differenza ontologica. La tecnica svela, infatti, possibilità che sono presenti ma nascoste nel mondo, ma lo fa provocando il mondo, chiamandolo perché si apra dinanzi al tecnico. «La tecnica è un modo del disvelare. La tecnica dispiega il suo essere [...] nell'ambito in cui accadono disvelare e disvelatezza $[\ldots]$, dove accade $[\ldots]$ la verità ${ }^{18}$. Lo stesso pensiero torna nelle lezioni e nella conferenza degli anni 1955-1956, Il principio di ragione, in cui l'autore mette in risalto le pretese della ragione soggettiva di conoscere il mondo secondo le proprie esigenze e possibilità, e non secondo ciò che il mondo potrebbe

\footnotetext{
${ }^{16}$ Heidegger aveva incontrato poco prima, nel 1935, Werner Heisenberg che, dopo le esperienze in fisica nucleare di de Brogli, aveva elaborato il principio d'indeterminazione. La necessità di costruire un discorso scientifico sulle chiare determinazioni reperibili sensibilmente non era più, quindi, assoluta: l'ideologia scientista è manifestata troppo sommaria. La tesi dell'incertezza poteva invece aprire un cammino di avvicinamento tra le nuove teorie scientifiche e la riflessione heideggeriana sulla differenza ontologica (Vedi M HEIDEGGER, «La questione della tecnica», p. 17, p. 21).

${ }_{17}$ M. HeIDEGGER, «La questione della tecnica», p. 5-27.

${ }^{18}$ M. HeIDEGGER, «La questione della tecnica», p. 10.
} 
mostrare di sé e da sé. Sembra che non appartenga più alla scienza il rispetto per le realtà in sé. «Dove e a chi deve essere reso il fondamento? La risposta è: deve essere reso di ritorno all'uomo, che determina gli oggetti in quanto oggetti nella modalità del rappresentare che giudica» $^{19}$. D'altronde, come afferma l'articolo «Che cosa significa pensare» del 1952, «la scienza non pensa» ${ }^{20}$, perché rinchiude le possibilità del sapere nei domini che considera alla sua disposizione e che può tecnicamente maneggiare.

La tonalità generale della riflessione di Heidegger non sembra quindi distaccarsi totalmente da quella di Husserl; l'uno e l'altro criticano la sottomissione della scienza alle possibilità offerte dai tecnici e dalla loro mentalità abbandonata all'ideologia del naturalismo oggettivistico. Heidegger aggiunge però alla riflessione di Husserl una possibile fondazione ontologica di questa dipendenza. Ecco il punto su cui i due filosofi si separano. Si erano già scontrati nel 1927, in occasione della proposta fatta dall'Encyclopaedia Britannica a Husserl di scrivere un articolo sulla «Fenomenologia». Husserl aveva domandato a Heidegger, il discepolo che preferiva per la sua intelligenza speculativa e la sua comprensione delle intenzioni della nuova fenomenologia, di leggere e di valutare una prima bozza di questo saggio. Il discepolo lo fece volentieri, aggiungendo però alcuni passi che non piacquero a Husserl. Siamo nello stesso anno della pubblicazione di Essere e tempo, che Heidegger dedicò al suo maestro, anche se entrambi dovevano già sapere che alcune differenze maggiori di dottrina vi si facevano vive.

Heidegger propose a Husserl una specie d'introduzione alla sua bozza. Si tratta di poche pagine che insistono sul ruolo della soggettività in filosofia; dopo il suo maestro, Heidegger evidenzia, infatti, l'importanza della "coscienza" in fenomenologia. Il discepolo introduce però il tema dell'ontologia che, conformemente alla tesi iniziale di Essere et tempo, sembra assumere la filosofia classica, almeno aristotelica e trascendentale; ecco ciò che dispiacque a Husserl. Secondo Heidegger, la filosofia fondamentale riflette sul massimamente universale, mentre le scienze particolarizzano i loro differenti domini, frammentando il campo della verità: «la totalità dell'ente rappresenta il campo nel quale le scienze positive della storia e, dello spazio trovano di volta in volta il loro ambito oggettuale» (D 61). L'idea filosofica di totalità ontologica permette di organizzare una gerarchia delle scienze secondo le loro

\footnotetext{
${ }^{19}$ M. HeIDEGGER, Il principio di ragione, p. 201, nella conferenza che ha lo stesso titolo del corso.

${ }^{20}$ M. HEIDEGGER, Saggi e discorsi, p. 88.
} 
particolarità, lasciando trasparire l'originalità dell'idea di totalità fuori dalla quale non si può pensare che ci sia qualcosa.

Nella tradizione filosofica che comincia con Parmenide, la riflessione sull'“ente" non trascura di coinvolgere il "pensare" o la «riflessione sul pensiero dell'ente» (D 62) ${ }^{21}$. Secondo Kant ugualmente, la problematica filosofica autentica «si muove nel campo della coscienza» (D 62). Heidegger precisa allora che la fenomenologia di Husserl si situa perfettamente in questa tradizione.

Il chiarimento fondamentale delle necessità del ritorno alla coscienza, la definizione radicale ed esplicita dell'itinerario e della legge di questo ritorno, la delimitazione di principio e la ricerca sistematica del campo della soggettività pura che si schiude con questo ritorno - tutto ciò è fenomenologia (D 62).

La considerazione della coscienza può però rimanere ambigua, fermarsi alla psicologia come a una «scienza positiva» (D 63) senza accedere però alla «soggettività trascendentale» (D 63). Sappiamo che la preoccupazione di Husserl era la stessa. «La psicologia pura, come scienza positiva della coscienza, rimanda alla scienza trascendentale della soggettività pura: questa è la realizzazione dell'idea della fenomenologia come filosofia scientifica» (D 63). La proposta introdotta da Heidegger considera poi la «psicologia pura» di cui determina l'oggetto, il metodo (la riduzione, l'eidetica) e infine la funzione fondamentale. In tutto ciò, l'autore segue le orme del suo maestro.

Queste pagine sono state scritte forse durante l'estate o all'inizio dell'autunno 27, quando Heidegger era ospite di Husserl per discutere, tra altri punti, dell'articolo proposto dall'Encyclopaedia Britannica. Dopo queste giornate di lavoro, Heidegger mandò il suo testo a Husserl, sapendo che aggiungeva qualcosa che non era stato previsto nella prima bozza del maestro; la novità consisteva precisamente nell'approfondimento della «questione della soggettività trascendentale e delle sue relazioni con la spiritualità pura» (D 74) nella prospettiva di una «completa determinazione» (D 77) della questione.

Una lettera di Heidegger a Husserl nel novembre 1927, inviata con alcuni allegati, era esplicita. Il primo allegato pone il problema in maniera precisa: la questione è quella dello statuto della categoria "mondo", la cui comprensione non può essere appoggiata su un ente intra-mondano. Heidegger concorda con Husserl su questo punto, ma rileva anche che, nel

\footnotetext{
${ }^{21}$ Nel poema di Parmenide (n. 8, v. 38), si legge che «è la stessa cosa pensare e pensare che è» (I presocratici. Testimonianze e frammenti, p. 276).
} 
testo del suo maestro, rimane indeterminata la riflessione su "chi" pensa il mondo, cioè su chi sta all'origine delle rappresentazioni delle cose del mondo. Per principio, chi sta all'origine di ogni rappresentazione non può cogliersi quale una di queste rappresentazioni senza rinchiudere l'argomento in un circolo vizioso. Si apre così la questione dell'ontologia che Heidegger presentava prima in un modo classico, ma che subisce adesso una torsione innovativa, ignorata da Husserl. «È proprio qui che sorge il problema: qual è il modo di essere dell'ente nel quale il "mondo" si costituisce?» (D 76). Heidegger indica allora che il suo Essere e tempo affronta il problema aprendo la questione dell'“esistere" poiché il "sé" nella sua effettività «non è mai solo semplicemente presente, ma esiste» (D 76).

Heidegger modifica così il senso husserliano della Erlebnis, dell'evento vissuto o della esperienza. Per Husserl, Erlebnis significa una struttura intenzionale immanente al vissuto, e meno l'esperienza in quanto vissuta da una persona singolare, e ancora meno quale apertura del Esserci all'essere. Il pensiero di Husserl va alle strutture, alle essenze, non al vissuto in quanto vissuto singolarmente. Per Heidegger invece, «le forme essenziali dei singoli Erlebnisse sono [...] di un Sé individuale, che esiste in base alle sue convinzioni, decisioni, abitudini, qualità caratteriali» (D 66). Essere e tempo asseriva che «l'Esserci non è soltanto un ente che si presenta fra altri enti. Onticamente, esso è piuttosto caratterizzato dal fatto che, per questo ente, nel suo essere, ne va di questo essere stesso» ${ }^{22}$.

Non dobbiamo intendere queste affermazioni di Heidegger alla luce di qualche esistenzialismo soggettivistico, nonostante una tradizione interpretativa di Essere e tempo che scartiamo con decisione. Notiamo che il titolo del libro del 1927 non nomina solamente 1'“essere", ma anche il "tempo", un tema che tormentava Husserl dall'inizio del secolo, nella prospettiva della coscienza e della sua estensione continua ${ }^{23}$. La differenza tra Husserl e Heidegger si manifesta però quando Heidegger parla nel primo allegato alla sua lettera del 22 ottobre 1927 di "fatticità": «la possibilità della costituzione trascendentale [...] è una possibilità centrale dell'esistenza del Sé nella sua effettività (das faktischen Selbst)» (D 76). L'esistenza, per Heidegger, non è solamente una presenza, ma viceversa una tensione o un dinamismo personale per il quale la "fatticità" è essenziale. Leggiamo nel $\S 4$ di Essere $e$ tempo queste righe:

\footnotetext{
${ }^{22}$ M. HEIDEGGER, Essere e tempo, p. 28.

${ }^{23}$ Del 1904-1905. Vedi E. HuSSERL, Per la fenomenologia della coscienza interna del tempo, $2001^{5}$.
} 
Quell'essere stesso verso cui l'Esserci può comportarsi in un modo o nell'altro e verso cui sempre in qualche modo si comporta, noi lo chiamiamo esistenza. [...] L'Esserci comprende sempre se stesso in base alla sua esistenza, cioè alla possibilità che gli è propria di essere e non essere se stesso. [... L'Esserci] è l'ente che, nel suo essere, già sempre si rapporta $a$ ciò a proposito del quale questo problema è posto» ${ }^{24}$.

L'esistenza umana è fatticità, cioè una esistenza gettata nel mondo in tal modo che essa sia in movimento, in-quieta (senza riposo), orientata fuori dal suo stato presente, existenza. L'«Introduzione» di Essere e tempo lo evidenza. Il $\S 1$ ricorda l'interesse della filosofia antica per il concetto più generale di tutti, il concetto di "ente"; il § 4 orienta la riflessione della filosofia classica sulla psychè quale punto d'ancoraggio della riflessione fondamentale sull'Esserci, sul Dasein. Questa decisione heideggeriana non induce a «una fallace soggettivizzazione della totalità dell'ente» ${ }^{25}$, ma al rispetto per la posizione esatta del problema ontologico.

Da ciò segue una differenza nella comprensione della "coscienza". Per Husserl, la coscienza è intenzionale e "costituente" (da ciò le critiche abituali al suo "soggettivismo" e alle sue difficoltà per pensare l'alterità dell'altra persona). Per Heidegger, la coscienza si situa invece in un contesto di chiamata paradossalmente silenziosa perché libera di sé, «una chiamata dell'Esserci di fronte alle proprie possibilità» ${ }^{26}$.

Heidegger aveva mandato poco prima a Husserl un esemplare di Essere e tempo con una dedica personale. Tuttavia Husserl non lesse con attenzione il volume prima dell'estate 29. Nel 1927 una lettura corsiva gli aveva permesso di riconoscere le linee maggiori del testo heideggeriano e i suoi orientamenti divergenti, ma senza speciali approfondimenti. In una lettera mandata a Roman Ingarden il 19 novembre 1927, Husserl riconosce che una distanza lo separa da Heidegger. Scrive che

mi dispiace che la sua [di Heidegger] opera e, probabilmente anche le sue lezioni, si presentino come qualcosa che per essenza è differente dalle mie opere e dalle mie lezioni [...]. Per la filosofia futura sarà molto importante se e come Heidegger si applicherà per cogliere le mie intuizioni universali (G 13).

\footnotetext{
${ }^{24}$ M. HeIDEGGER, Essere e tempo, § 4, p. 28, p. 29, p. 31.

${ }^{25}$ M. HEIDEGGER, Essere e tempo, \$ 4, p. 31.

${ }^{26}$ M. HEIDEGGER, Essere e tempo, § 56, p. 332,
} 
Questa lettera termina con la speranza che l'intelligenza di Heidegger gli permetterà di meglio percepire la posta in gioco della fenomenologia autenticamente husserliana. Un mese dopo però, il 26 dicembre, Husserl scrisse a Ingarden che «Heidegger non ha colto il senso di questo cammino e così il senso complessivo del metodo della riduzione» (G 13). Sarà forse durante questo mese che Husserl avrà fatto la sua lettura corsiva di Essere e tempo.

Le sue prime impressioni furono confermate durante una lettura più attenta, nel 1929, forse segnata dall'amarezza nata delle difficoltà incontrate con il discepolo negli anni recenti. I giudizi di Husserl su Heidegger si faranno poi, anno dopo anno, sempre più pesanti. Dirà che la proposta heideggeriana non è «scientifica»; egli stesso non può sopportare «l'“abissalità" dell'“irrazionalismo heideggeriano", la cui "oscura mistica dell'esistenza" nasconde "il pericolo più grande" per la fenomenologia» (G 14). Il 1929 sembra essere l'anno della definitiva presa di distanza di Heidegger; la sua conferenza «Che cos'è la metafisica» ${ }^{27}$ non nomina, infatti, né Husserl né la fenomenologia, ed Otto Pöggeler definisce tale «congedo [di Heidegger] alla fenomenologia» (vedi D 56, n. 78).

Riassumiamo la situazione. Husserl critica Heidegger perché questi andrebbe da una riflessione strutturale, universale, fedele alle esigenze della riduzione, a un'antropologia in cui appare centrale l'esistenza individuale; Heidegger critica invece Husserl perché non distinguerebbe "ente" ed "essere", senza parlare della distinzione tra "essere" ed "esistere" di cui tratta l'inizio del corso I problemi fondamentali della fenomenologia del semestre estivo del $1927^{28}$. La seconda lettera a Ingarden segnala però che il problema fondamentale è quello della "riduzione", applicata da Heidegger in un modo che Husserl non aveva previsto. Siamo quindi giunti alla questione essenziale della riduzione che caratterizza la riflessione filosofica quando considera gli sforzi della ragione nel lavoro scientifico.

La filosofia, classicamente, pone alle scienze la questione del loro fondamento razionale. L'idea di fondamento potrebbe però essere intesa in un modo sbagliato se significasse che la filosofia avrebbe la possibilità e il dovere di imporre alle scienze i principi dei loro specifici procedimenti. Già per Aristotele, non si può esibire una simile esigenza. Neanche la modernità avrebbe avanzato tale pretesa. Per esempio, quando Cartesio propose la

\footnotetext{
${ }^{27}$ M. HeIDEGGER, «Che cos'è la metafisica?», p. 59-77.

${ }^{28}$ Vedi M. HeIDEgGER, I problemi fondamentali della fenomenologia, il § 8. Il titolo del libro costituisce verosimilmente un plagio ironico del titolo dato da Husserl alle sue lezioni dell'anno accademico 1904-1905, semestre invernale: I problemi fondamentali della fenomenologia. Lezioni sul concetto naturale di mondo, 2008 (titolo della pubblicazione tedesca: Aus den Vorlessungen Grundprobleme der Phänomenologie).
}

Sapere aude - Belo Horizonte, v. 7 - n. 12, p. 301-329, Jan./Jun. 2016 - ISSN: 2177-6342 
sua metafora dell'albero ${ }^{29}$ per illustrare una possibile organizzazione delle scienze, non avrebbe pensato che la filosofia possa imporre alle scienze i principi delle loro dimostrazioni. La metafisica alle radici dell'albero delle scienze offre solo un modello di certezza, non di dimostrazione in tutti i campi; sarebbe invece la geometria che offrirebbe lo schema migliore di procedimento argomentativo universale, anche in metafisica ${ }^{30}$.

Husserl analizza il problema alla luce della tesi dell'intenzionalità fenomenologica e del metodo di riduzione a un Erlebnis d'ordine epistemologico, formale, intelligibile per gli scienziati. Heidegger considera invece i concetti fondamentali delle scienze ${ }^{31}$ che traggono il loro senso dalle dinamiche evidenziate dalla riflessione sulla differenza ontologica, un modo d'approfondimento della struttura dell'intenzionalità trasportata in ontologia. L'integrazione delle scienze nella filosofia consiste nell'assegnare loro un sito in una gerarchia di atti conoscitivi, organizzati con criteri che non vengono dai loro oggetti specifici, ma dall'apertura delle domande che vi sono attuate a partire da situazioni particolari. In fenomenologia, l'intenzionalità ontologica costituisce il criterio fondamentale che ordina le scienze, e la riduzione è il metodo che pone in luce l'origine delle intenzioni esercitate nei differenti campi della ragione. Abbiamo visto che Husserl criticava Heidegger precisamente sul punto della riduzione; Heidegger, dando all'intenzionalità una comprensione ontologica che Husserl non considerava, non poteva non cambiare il senso stesso della riduzione, che diviene una ermeneutica o una analitica esistenziale.

La "riduzione fenomenologica" è stata tematizzata per la prima volta da Husserl nel 1907, nelle cinque conferenze di Göttingen. La prima tappa della riduzione è l'epoché di ogni trascendenza, cioè la messa fra parentesi di tutte le nostre comprensioni spontanee del mondo così come della convinzione che il conosciuto esista in qualche modo e che questa sua esistenza sia una determinazione essenziale per la nostra conoscenza. L'epoché è già una riduzione, negativa in qualche modo, purificatrice comunque; consiste, infatti, in una operazione mentale che capovolge le presupposizioni delle scienze empiriche per ritrovare l'intenzione originaria della coscienza. Una buona conoscenza per gli scienziati è positiva e di essenze; ad essa non importa esageratamente l'esistenza delle cose singole. La fenomenologia

\footnotetext{
${ }^{29}$ Vedi sopra R. DESCARTES, «Lettera dell'Autore a colui che ha tradotto il libro» (cf. sopra, nota 6).

${ }^{30}$ L'espressione "More geometrico" s'incontra nelle risposte alla seconda mediazione - vedi R. DESCARTES, Opere, $1637-1649$, p. 892.

31 «Poiché ognuno [degli ambiti delle scienze] può esser tratto esclusivamente da un dominio dell'ente, [l'indagine] preliminare che istituisce i concetti fondamentali è null'altro che l'interpretazione di questo ente rispetto alla costituzione fondamentale del suo essere» (M. HEIDEGGER, Essere e tempo, § 3, p. 26).
} 
husserliana si vuole similmente "eidetica", e la sua pretesa d'universalità trae da ciò la sua legittimità scientifica:

\begin{abstract}
Almeno per chi possa mettersi nella posizione del puro guardare, e tenere lontana da sé ogni pre-opinione naturale, è più facile riconoscere che non solo delle individualità, ma anche delle universalità, cioè oggetti universali e stati di cose universali, possono pervenire ad assoluta datità diretta. Questo riconoscimento è d'importanza decisiva per la possibilità della fenomenologia ${ }^{32}$.
\end{abstract}

Ecco il punto per cui, nel 1927, Husserl criticava Heidegger; le sue prospettive sarebbero troppo esistenziali, cioè individuali, e cambierebbero così il senso dell'intenzionalità e della riduzione. Il vissuto ridotto alla sua essenza è, per Husserl, un eidos universale e scientifico, per esempio il rosso intuito come tale ${ }^{33}$, una operazione mentale come un calcolo aritmetico. Tutti gli "oggetti" di conoscenza che sono universalmente accessibili e cui non importa che corrisponda o no alcun oggetto sensibile sono orizzonti d'intenzionalità fenomenologica. Sottomettendosi a questa condizione d'assoluta universalità, la fenomenologia è scientifica in un modo eminente. Husserl lo conferma nell'articolo dell'Encyclopaedia Britannica:

L'epoché universale riguardo a quel mondo che diventa presente alla coscienza (la sua "messa fra parentesi") esclude dal campo fenomenologico il mondo semplicemente essente per il soggetto in questione, e al suo posto subentra il mondo presente alla coscienza in maniera determinata "come tale", il "mondo fra parentesi" (il mondo percepito, ricordato, giudicato, pensato, valutato ecc.) o, il che è lo stesso, al posto del mondo (ovvero della singola cosa meramente mondana) subentra il singolo senso della coscienza nei suoi diversi modi (senso della percezione, senso del ricordo ecc.) (D $\left.86^{34}\right)$.

L'intenzionalità husserliana è trasformata da Heidegger in questione ontologica ${ }^{35}$. Segue un abbandono della riduzione eidetica, a favore di una ermeneutica esistenziale, di una analitica del Esserci, in cui Husserl vede una modifica della sua fenomenologia. Heidegger critica quindi l'intenzionalità husserliana e non si preoccupa più della riduzione proposta dal suo maestro.

\footnotetext{
${ }^{32}$ E. HUSSERL, L'idea della fenomenologia, p. 87.

${ }^{33}$ Vedi E. HuSSERL, L'idea della fenomenologia, p. 93.

34 Questo passo potrebbe commentare alcune righe delle Idee I: «tutto ciò che si dà originalmente nell" "intuizione" (per così dire in carne e ossa) è da assumere come esso si dà, ma anche soltanto nei limiti in cui si dà» (E. HUSSERL, Idee per una fenomenologia pura e per una filosofia fenomenologica, p. 52-53).

${ }^{35}$ Vedi nota 23.
}

Sapere aude - Belo Horizonte, v. 7 - n. 12, p. 301-329, Jan./Jun. 2016 - ISSN: 2177-6342 


\section{Michel Henry e la riduzione}

Tra gli autori che hanno criticato Husserl, Michel Henry sembra essere uno dei più radicali. Lo è stato nel suo libro del 2000, Incarnazione ${ }^{36}$, ma già in altri testi, in particolare in un articolo pubblicato nel 1991 in occasione di una discussione dell'allora recente libro di Jean-Luc Marion, Donazione e riduzione ${ }^{37}$. Nell'articolo del 1991, «Quatre principes», Henry evidenzia i massimi principi della fenomenologia, i primi tre essendo di Husserl e il quarto di Marion. Ecco il primo: «tanta apparenza, quanto essere». A questa formula, Henry ne preferisce un'altra: «tanto apparire, quanto essere»; con il termine "apparire” (un verbo) al posto di "apparenza" (un sostantivo), si evita, infatti, l'ambiguità delle culture per le quali le apparenze sono illusioni o inganni. Per la fenomenologia, non si può sostenere che l'apparire illuda; vedremo perché. Il secondo principio husserliano approfondisce il primo: «ogni intuizione originalmente offerente è una sorgente legittima di conoscenza» ${ }^{38}$. Questo principio dovrà essere spiegato con un'attenzione particolare a un aspetto importante: l'apparire è determinato dal suo modo d'apparire, dal suo "come", e dai suoi "limiti"39. Il terzo principio esprime il fondamento fenomenologico dei due precedenti, l'intenzionalità: andare «diritto alla cosa stessa». Quanto al quarto principio, che Marion propone nel suo libro del 1990, esso tocca direttamente la questione della riduzione: «tanto più riduzione, quanto più donazione», una formulazione che Henry adotta.

La critica di Henry al primo principio considera una incoerenza che vi sarebbe immanente (e che, insomma, il cambiamento dei sostantivi in verbi non sembra modificare essenzialmente $\left.^{40}\right)$. Da una parte, infatti, si evidenzia una identità tra "apparire" ed "essere". È ovvio che l'apparire è di un essere che appare. «È perché l'apparire spiega il suo regno che l'essere spiega pure il suo, perché hanno un solo e medesimo regno, una sola e medesima essenza» (Q 4). Se non ci fosse "un” essere, come ci sarebbe un "apparire”? Questa identità

\footnotetext{
${ }^{36}$ M. HENRY, Incarnazione: una filosofia della carne, 2001. Le pagine 32-35 (nel $\S 2$ del libro) riprendono quasi testualmente i passi più importanti di Q.

${ }^{37}$ J.-L. MARION, Riduzione e donazione. Ricerche su Husserl, Heidegger e la fenomenologia, 2010. L'originale in francese è del 1989.

${ }^{38}$ E. HUSSERL, Idee per una fenomenologia pura, $\S 24$, p. 52.

${ }^{39}$ In tedesco: als was is sich gibt (come si dà) e Schranken (limiti).

40 Potremmo quindi interrogarci sull'opportunità di un simile cambiamento: serve in realtà a radicalizzare la critica, a spostare la riflessione sulle "rappresentazioni" verso le considerazioni sugli "atti", ciò che importerà per pensare la "vita" con cui Henry nomina l'originario assoluto. Perché però parlare di vita, un sostantivo, piuttosto che di "vivere", un verbo? Lo stile retorico di Henry è brillante, ma ugualmente molto oscuro.
} 
evidente non ha però una massima forza nella tradizione filosofica. "Apparire" e "essere", infatti, «non stanno sullo stesso piano, la loro dignità ontologica $[\ldots]$ non è la stessa» (Q 4). Per il platonismo comune, l'apparenza non “è", “è” solamente l'essere; ogni apparenza deve rimandare a un essere originario e fondatore. Tuttavia, la riflessione invita a rovesciare quest'ordine di distinzione ontologica. Per Henry, non c'è “essere" se non c'è "apparire". Contrariamente quindi a ciò che affermano i manuali, è l'apparire che fonda per noi l'essere. L'essere è nulla se non appare. L'essenza dell'essere sta dunque nell'apparenza, la cui essenza è propriamente di apparire (il verbo!), punto. L'ontologia s'identifica perciò con la fenomenologia; è fenomenologia dell'apparire. Henry trae a questo riguardo un modello di riflessione dalle osservazioni di Cartesio. Per questi, la cogitatio manifesta il proprio essere nell'atto stesso di manifestarsi. Cartesio scrive nelle sue Meditazioni: «Ma che cosa sono? Una cosa pensante» ${ }^{41}$. L'essere del pensare è il pensare fenomenicamente. Poiché l'apparire effettivo è la condizione ontologica dell'essere pensante, l'esperienza riflessiva cartesiana serve a Henry da "campione" per interpretare il compito della fenomenologia.

La critica del dualismo platonico conduce direttamente alla critica del terzo principio: «diritto alla cosa stessa» (ci volgeremo più tardi al secondo principio, spiegando il perché di questa inversione). L'analisi del primo principio ha ridotto l'essere all'apparire. Ora il terzo principio suppone una differenza tra la "cosa" (Sach - non importa che sia "essere" o “apparire") e il movimento che vi porta $(z u)$. La problematica è quella dell'intenzionalità, che Henry criticherà frontalmente più tardi ${ }^{42}$. La critica sorge adesso dalla riformulazione del primo principio. Se dobbiamo, infatti, fare uno sforzo quando tendiamo verso le cose, è perché le cose non ci appaiono chiaramente, o perché non ci fidiamo delle apparenze, o perché ci sembra che ci siano delle ragioni per distinguere essere e apparire. Abbiamo però visto che non c'è "apparire" se non c'è "essere"; l'essere è ciò che sta nel proprio apparire; essere è apparire. Il terzo principio quindi, quello dell'intenzionalità per cui la "cosa" è " $z u$ " $\mathrm{o}$ a distanza, è da giudicare anti-fenomenologico.

Il pensiero classico è però più sottile. Sostiene che c'è un legame tra la cosa e i mezzi utili per raggiungerla. Dirà anzi che i mezzi determinano le cose mirate, e che le cose mirate si

\footnotetext{
${ }^{41}$ R. DESCARTES, Opere, 1637-1649, p. 719, nella Meditazione seconda. Al testo di Cartesio, Henry aggiunge: «la cui essenza è interamente di pensare» (Q 5). Sappiamo poi quanto questa tesi impone una domanda strana: quando non penso, sono? La stranezza di questa domanda non cambia niente, comunque, all'incontrovertibilità dell'affermazione: cogito, sum, «ogni volta» che sono in attività di pensare, dice Cartesio (p. 715).

${ }^{42}$ Vedi M. HENRY, «Phénoménologie non intentionnelle: une tâche de la phénoménologie à venir», p. 383-397.
}

Sapere aude - Belo Horizonte, v. 7 - n. 12, p. 301-329, Jan./Jun. 2016 - ISSN: 2177-6342 
lasciano così toccare. Questo pensiero classico, che media le apparenze e le realtà con il metodo, è confermato dall'interpretazione contemporanea che riconosce la progressività della conoscenza scientifica. Notiamo che questa progressività è possibile perché conoscere qualcosa non fa essere questa cosa, che è prima di essere conosciuta e che può quindi essere sempre meglio conosciuta con mezzi d'avvicinamento più perfezionati. «Ciò che si dà nel fenomeno, si dona [...] prima che l'apparire lo istalla in questa condizione di ciò che appare, di ciò che è quale fenomeno per noi» (Q 7). Questa anteriorità fa sì che le teorie scientifiche si susseguono senza essere erronee ma solamente approssimative e verosimilmente sempre più precise, realistiche. Il problema, su cui la tradizione classica ha sempre fondato la sua distinzione tra la sostanza e l'accidente, dice però che prima di apparire, ciò che si dona non appare. Henry riconosce in questa tesi il platonismo comune che abbiamo già notato in agguato nel primo principio, ma che non regge in alcun modo. La realtà appare in ogni apparenza, è le sue apparenze. La scienza ha quindi, in ogni momento del suo sviluppo, un valore veritativo.

L'autore illustra la sua difficoltà teoretica con una metafora tratta dall'esperienza della luce. Supponiamo che "essere" sia come la luce. Se da una parte la luce è indifferente «a tutto ciò che illumina» $\left(\mathrm{Q} 8^{43}\right)$, e se dall'altra il fenomeno deve venire alla luce, il fenomeno diviene ugualmente indifferente. Avverrebbe, infatti, in un ambito d'indifferenza, perdendo ogni forma. La fenomenologia che si appoggia su questa metafora si autodistrugge. Essendo l'origine indifferente, lo è anche l'originato; non c'è niente verso cui orientare $(z u)$ lo sguardo a partire dal fenomeno se in esso non c'è niente (sach) che appare. L'intenzionalità fenomenologica non tende verso 1'“essere" universale che, indeterminato, sarebbe dietro il fenomeno determinato. «Andare alla cosa stessa» non significa andare più profondamente dell'apparenza; significa cogliere l'apparire dell'apparenza.

Il secondo principio (l'intuizione) può essere commentato adesso. Come abbiamo visto con Husserl, questo principio situa ogni universalità nell'orizzonte dell'intuizione fenomenologica. Accogliamo però ciò che s'intuisce «come si dona», cioè in una maniera particolare, in «carne e ossa» (l'espressione è di Husserl ${ }^{44}$ ). C'è infatti una varietà d'intenzioni che tendono verso ciò che si determina apparendo. Le nostre intenzioni sono molte, sensibili,

\footnotetext{
43 «L'umana sapienza, che resta sempre unica e medesima per quanto applicata a differenti oggetti, e non riceve da essi una maggiore distinzione di quella che la luce del Sole riceve dalla varietà delle cose che illumina [...]» (nella prima delle Regole per la direzione dell'ingegno, in R. DESCARTES, Opere postume, 1650-2009, p. 685).

${ }^{44}$ Vedi per esempio qui sopra, la nota 35.
} 
percettibili, razionali ecc. Ecco la seconda ragione per cui, secondo Henry (la prima ragione, radicale, è che la tesi husserliana distingue "essere" e "apparire"), l'origine universale o l'essere che si presenta idealmente in molti modi «non è né presentato né dato, ma viceversa scartato, o per meglio dire abolito» (Q 12) dal principio dell'intuizione che sottomette l'intenzionalità alla particolarità di ogni apparire. La fenomenologia si è infatti sviluppata dopo Husserl in molti tipi di fenomenologia più meno "eretici” grazie all'ambiguità che il suo fondatore aveva proposto dell'intuizione fenomenologica.

Veniamo adesso alla questione della scienza. Non dobbiamo dire che le scienze esercitano o adoperano intenzioni specifiche, particolari, ciascuna essendo determinata da una delle nostre capacità conoscitive? La scienza, opera umana, è intenzionale. Ora sembra che la tesi husserliana sulla fenomenologia e la sua critica della scienza empirica ignori questa determinazione. $\mathrm{O}$ più esattamente, Husserl intende mostrare che la tecno-scienza non porta l'intenzione della conoscenza scientifica in generale al suo compimento. In realtà, Husserl non nega che la scienza abbia una validità razionale ma nega ogni valore all'interpretazione empirista della scienza e all'ideologia che impone di considerare "scientifico" solamente ciò che corrisponde a questa ideologia. L'ideologia scientista è solo una interpretazione sbagliata, che non considera la reale dinamica delle scienze e dei loro metodi. Non dovremmo però interpretare fenomenologicamente questa pretesa della ragione ed evidenziare il suo senso? O dovremo dire che non ha alcun senso? Ma se non ha alcun senso, le prodezze della tecnica non saranno espulse dal dominio del senso? Che cosa diverrebbe allora il loro "apparire"?

Henry non si preoccupa più di Husserl della fondazione dell'empirismo. S'inquieta piuttosto della riduzione della scienza alla tecnica e l'abisso che si è creato in Occidente tra la cultura e le scienze ${ }^{45}$. Il filosofo di Montpellier pensa poi che sia più utile approfondire le critiche già elaborate da Husserl e Heidegger riguardo alla tecno-scienza e correggere i loro principi di fenomenologia. Potrà forse rendere così più decisiva la loro contestazione della mentalità contemporanea. Dobbiamo quindi porre di nuovo la questione dell'evidenza dell'oggettività scientifica. Quest'apparente evidenza non è adeguatamente scomposta dai principi di Husserl che abbiamo esaminato. Bisogna denunciare prima di tutto la pretesa delle scienze che distruggono la vita con gli strumenti della loro potenza tecnica. Il libro pubblicato da Henry nel 1987, La barbarie, evidenzia la forma matematica delle scienze contemporanee,

\footnotetext{
${ }^{45}$ Vedi M. HENRY, La barbarie, p. 13-42.
} 
una eredità di Galileo Galilei che, dell'esperienza sensibile, non può e non vuole conoscere niente perché elabora scientificamente solo le esperienze che sono messe in forma da principi matematici apriori ed evidenti, e dunque non criticabili ${ }^{46}$. La scienza, in realtà idealista e costruttivista, si allontana dalle realtà concrete e sensibili. La scienza matematizzata ignora ogni realtà, ignora la vita. Quando si sottomette poi all'impulso della tecnica, non s'interessa più dei viventi ma solo dell'autosviluppo e dell'efficienza dei suoi strumenti.

La critica di Henry alla scienza contemporanea si fonda su una critica radicale dell'intenzionalità. Il termine "intenzionalità" indica una tensione verso qualche trascendenza, qualche alterità; evoca cioè un esilio fuori di se stesso, e quindi un'alienazione. L'intenzionalità razionale, che tende verso ciò che è ma che non lo può senza le mediazioni concettuali matematizzate, manca la realtà stessa ed è ingannevole. Le rappresentazioni non sono presenze ma immagini e strutturazioni della realtà; possiamo quindi "giocare" con esse, anche se con regole scientificamente determinate. L'intenzione fenomenologica, di cui abbiamo evidenziato la particolarità, è alla base di ogni manipolazione delle realtà singole perché offusca il senso del presente, dell'immediatamente dato, del sensibile, del vissuto. Essa espelle la vita, la nasconde dietro i suoi calcoli. La scienza contemporanea, compresa la biologia, lascia la vita dietro di sé, manifestando così che la sua intenzione non è realista. I positivisti dicono che la vera scienza assume il sensibile. Per Henry, come per Heidegger, essi e i loro progetti tecnici non sanno niente del sensibile. La fenomenologia, se non corregge subito la sua comprensione dell'intuizione intenzionale, potrebbe cadere nella stessa ignoranza.

Il positivismo, che di positivo conosce solo ciò che la ragione pone di fronte a sé, cioè le sue rappresentazioni calcolate, ha rovinato la cultura, o piuttosto ha imposto una cultura alienante. Un articolo del 1989, che compatta il libro del 1987, afferma che «l'alienazione dell'essere umano non è mai stata talmente completa [come oggi], se essere alienato significa essere divenuto straniero a se stesso» ${ }^{47}$. Una prova di questa alienazione si dimostra in etica. La tecnica, infatti, è capace di fabbricare la bomba atomica e di liberare tutta la sua potenza, di distruggere l'umanità. Essa però non potrà mai dire da sola quando e dove conviene utilizzarla. Il problema tralasciato dalla tecno-scienza è quello del senso o della finalità del

\footnotetext{
46 «Rinunciando alla corrispondenza esatta con la piena concretezza dell'oggetto e, prima di tutto, con il suo modo di donazione, [la scienza] si propone quale assioma metodologico di ritenere solamente dell'oggetto, del "donato", ciò che essa pensa essere capace di discutere» (M. HENRY, La barbarie, p. 135).

${ }^{47}$ M. HENRY, «Ce que la science ne sait pas» p. 423.
} 
nostro sapere; la tecnica rimane silenziosa sulla propria finalità e impedisce alla scienza che si sottomette a essa di fare chiarezza su questo punto.

La critica filosofica della cultura tecnica non si accontenta però di una postura etica, considerando solamente il "dover essere". Una critica teoretica dell' “essere" è indispensabile. La critica dell'intenzionalità husserliana e l'approfondimento della riduzione la permettono. La "riduzione" costituisce una linea metodologica che mira l'uno, che sia eidos o principio originale; è sempre, in un modo o nell'altro, una reductio ad unum. La "Sach" radicale, la "cosa" mirata intenzionalmente, non è prima di tutto "positiva" e "oggettivabile", ma una presenza immediata a se stesso, il cui modello è la "vita". L'esperienza riflessiva cartesiana partecipa di questa esigenza. La cogitatio ne è il modo più conosciuto: «cogito, sum». Ne è un altro il riconoscimento della presenza a sé quando, durante un sogno notturno, qualche paura nasce in me e "mi" risveglia ${ }^{48}$. Il «sapere primitivo della vita, per cui essa non fa altro di sapere se stessa e non conosce altro di sé, sta operando nei nostri comportamenti più elementari, in ogni saper-fare, in ogni azione, in ogni prassi» ${ }^{49}$. La scienza oggettiva non sa niente di questa vita spirituale, che la «precede perché è la sua condizione inosservata e tuttavia imprescindibile» ${ }^{50}$.

Una riflessione sulla riduzione fenomenologica non potrebbe essere adesso di aiuto? Il libro di Jean-Luc Marion, Riduzione e donazione, che è stato all'origine dell'articolo di Henry sui «Quattro principi», è certamente uno dei testi che hanno segnato di più la storia recente della riduzione fenomenologica. Nel secondo capitolo di questo libro, Marion attribuisce due sensi alla riduzione. Un primo senso è propriamente husserliano. L'obiettivo è qui di ordine epistemologico, fondatore del senso della scientificità piuttosto che dell'oggettività empirica; il suo cammino è trascendentale.

Per Husserl il fenomeno ridotto nella sua più evidente presenza offre solo un correlato (noematico) alla coscienza; non appare mai come un ente, a sua volta suscettibile di rinviare non solo alla coscienza (idealismo trascendentale), ma al senso dell'essere ${ }^{51}$.

\footnotetext{
${ }^{48}$ Vedi di M. HenRY, «Une approche phénoménologique du christianisme» p. 351, che rimanda alle Passioni dell'anima di Cartesio, § 24, un esempio assai ricorrente nei testi di Henry.

${ }^{49}$ M. HENRY, «Ce que la science ne sait pas», p. 426.

${ }^{50}$ M. HENRY, «Ce que la science ne sait pas», p. 426.

${ }^{51}$ J.-L. MARION, Riduzione e donazione, p. 114.
}

Sapere aude - Belo Horizonte, v. 7 - n. 12, p. 301-329, Jan./Jun. 2016 - ISSN: 2177-6342 
Un secondo senso è evidenziato analizzando un testo di Heidegger ${ }^{52}$; coincide con la critica del primo principio della fenomenologia proposta da Henry: tra l'apparire e l'essere, non c'è distanza.

\begin{abstract}
L'interpretazione non consiste nel vedere un altro ente, ma a vedere in un altro modo l'ente. Tuttavia, non si tratta di vederlo, per la prima volta, come un ente, ossia come scoperto nella presenza, in breve di vederlo in quanto fenomeno. Il fenomeno appare: esso deriva dalla sua propria uscita dall'inapparenza verso l'apparizione evidente; vederlo in tal modo significa perciò vederlo nella misura in cui non cessa di apparire, cioè di liberarsi dall’inapparenza per fissarsi nella presenz ${ }^{53}$.
\end{abstract}

In altre parole, la fenomenologia è sapere dell'epistēmē e sapere della fenomenalità. Sotto questo ultimo punto di vista, le critiche di Husserl a Heidegger non sono totalmente errate; l'autore di Essere e tempo, sostituendo l'ermeneutica alla riduzione, torna infatti all'ontologia per la quale gli enti sono da differenziare; l'Esserci è particolare, ma è anche "ente" "54. Il quarto principio, «tanto più riduzione, quanto più donazione» (Marion), radicalizza la posizione heideggeriana, accettando le sfide eidetiche di Husserl. L'idea di donazione viene dalla pratica estrema della riduzione per cui «ciò che appare nell'apparire è prima e necessariamente l'apparire stesso» (Q 17). Il sapere interiore dell'Esserci rimane normativo. È il sapere dell'estraneità della coscienza dal mondo degli enti. L'Esserci si sa differente dagli enti, e questo sapere sostiene la critica di qualsiasi ontologia, heideggeriana o altra. Con Husserl e contro Heidegger, Marion mette alla presenza di una «subordinazione dell'ontologia alla fenomenologia» (Q 19). Il metodo della riflessione è quello della riduzione o, per meglio dire, di un «regresso» (Q 19) che evidenzia l'eccesso dell'originario che sta più in alto di ogni concezione. La coscienza è ascolto di una chiamata, ma senza voce; è ascolto di un semplice atto di donazione, vero fenomeno originario.

Henry rileva che si può andare poi in direzione della tesi dell'autodonazione della vita e della sua automanifestazione ${ }^{55}$. La fenomenologia di Marion, ispirata ovviamente a Emmanuel Levinas, decostruisce l'ontologia e rivela la radicale finitezza di ogni «orizzonte statico» (Q 24). La riflessione non si vede però condannata a un'immanenza chiusa in sé:

\footnotetext{
${ }^{52}$ M. HeIDEGGER, Prolegomeni alla storia dl concetto del tempo, 1991.

${ }^{53}$ J.-L. MARION, Riduzione e donazione, p. 109-110.

${ }^{54}$ Vedi Q 22.

${ }^{55}$ La letteratura odierna su questo punto è immensa. Vedi per esempio C. CANULlO, La fenomenologia rovesciata. Percorsi tentati in Jean-Luc Marion, Michel Henry e Jean-Louis Chrétien, 2004, p. 140-175; A.P. VIOLA, Dal corpo alla carne, 2005; Y. MEESSEN, Percée de l'ego. Maître Eckart en phénoménologie, 2016.
} 
Congedare l'essere è possibile soltanto fenomenologicamente. Congedare l'essere è possibile soltanto se, ogni fenomenalità essendo assente e nonostante questa assenza, qualcosa è ancora possibile piuttosto di niente - qualcosa, vale a dire l'arcirivelazione della vita (Q 24).

Dobbiamo però fermarci adesso. Per uno studio dell'approfondimento henryano della riduzione, si dovrebbero leggere alcune pagine di Incarnazione ${ }^{56}$.

\section{CONCLUSIONE}

La domanda dei fenomenologi, con Husserl e dopo di lui, è costante: può la fenomenologia essere non solamente una scienza rigorosa ma la scienza più rigorosa di tutte? Il concetto di "rigore" implica il riconoscimento di un metodo che sia conveniente. La convenienza del metodo dipende poi dalla finalità che è perseguita. La finalità qui in gioco è quella che motiva lo sviluppo della scienza, cioè una conoscenza completa e definitiva. Ora non c'è scienza completa senza conoscente. L'auto-implicazione del conoscente è costitutiva del metodo filosofico, che si presenta quindi quale scienza più rigorosa alla condizione che la soggettività del conoscente non sia oggettivata e che l'oggettività scientifica sia rispettata. L'inchiesta riguardante il principio più universale è perciò di ordine trascendentale: ciò che il sapere intende raggiungere è interiore al movimento che vi tende ma non l'origine di questo movimento. La riduzione percorre quindi un cammino di purificazione e invita a riconoscere ciò che anima la ricerca intellettuale, cioè il senso della ricerca per la coscienza (Husserl), l'offerta di senso nei fenomeni che ci vengono incontro (Heidegger), l'integrazione della ricerca nella vita (Henry).

Notiamo però che i fenomenologi non integrano le proposte oggettivanti delle scienze contemporanee; anzi, il loro atteggiamento è decostruttivo delle virtù che la cultura comune attribuisce oggi all'ideologia empirista, e poi decostruttivo di ogni ontologia. Si pone perciò questa domanda: la fenomenologia potrà ispirare un rinnovamento delle scienze contemporanee, senza ignorare i loro risultati dai punti di vista scientifici (conoscenza della realtà del mondo e dell'uomo) e tecnici (pratica che maneggia il mondo perché corrisponda alla capacità che ha l'uomo di tendere verso una vita migliora)? Tra la tecno-scienza e la

${ }^{56}$ M. HENRY, Incarnazione, § 2, p. 30-36.

Sapere aude - Belo Horizonte, v. 7 - n. 12, p. 301-329, Jan./Jun. 2016 - ISSN: 2177-6342 
fenomenologia, sembra che ci sia adesso un abisso.

Come pensare questa distanza? Abbandonando la tecno-scienza al suo destino e riservando per sé un mondo astratto di senso? O condannando la tecno-scienza con un richiamo estrinseco all'etica, lottando quindi contro l'atteggiamento autosufficiente della scienza empirica odierna? Gli autori che abbiamo commentato sanno che una critica etica della tecno-scienza non basta, che la critica deve essere teoretica per essere radicale. Se però la critica teoretica non integra la scienza ma espelle da sé ogni riconoscimento della sua potenza effettiva sul mondo, sarà una critica soddisfacente che avrà un'incidenza culturalmente utile? Non dobbiamo ricostruire un'ontologia? O il compito della riflessione non sarà di richiamare all'attenzione su un "tutto" di cui la "coscienza" è una parte distinta ma essenziale, la cui originalità condiziona il buon funzionamento delle altre?

\section{BIBLIOGRAFIA}

BANCALARI, St. Logica dell'epochè. Per una introduzione alla fenomenologia della religione. Pisa, ETS (Philosophica), 2015, pp. 135-155.

CANULLO, C. La fenomenologia rovesciata. Percorsi tentati in Jean-Luc Marion, Michel Henry e Jean-Louis Chrétien, Rosenberg \& Sellier (Dubbio \& speranza), Torino 2004.

DESCARTES, R. Opere postume, 1650-2009. trad. G. Belgioioso, Bompiani [Il pensiero occidentale], Milano 2009.

DESCARTES, R. Principi della filosofia. In: Opere, 1637-1649. A cura di G. Belgioioso, Bompiani [Il pensiero occidentale], Milano 2009.

DILTHEY, W. I tipi di visione del mondo e la loro formazione nei sistemi metafisici. In: Scritti filosofici (1905-1911). A cura di P. Rossi, UTET (Classici della filosofia), Torino 2004.

HEIDEGGER, M. «Che cos’è la metafisica?». In: Segnavia, trad. di F. Volpi, Adelphi (Biblioteca filosofica), Milano 2002, p. 59-77.

HEIDEGGER, M. Essere e tempo, trad. di P. Chiodi, Longanesi (I marmi), Milano $1976^{6}$.

HEIDEGGER, M. I problemi fondamentali della fenomenologia, trad. di A. Fabris, Il melangolo (Opera), Genova 1990.

HEIDEGGER, M. Il principio di ragione, trad. di G. Curisatti e F. Volpi, Adelphi (Biblioteca filosofica), Milano 1991. 
HEIDEGGER, M. «L'epoca dell'immagine del mondo». In: Sentieri interrotti, trad. di P. Chiodi, La nuova Italia (Strumenti), Firenze 1984, pp. 71-101.

HEIDEGGER, M. «La questione della tecnica». In: Saggi e discorsi, trad. di G. Vattimo, Mursia [Biblioteca di filosofia], Milano 1976.

HEIDEGGER, M. Prolegomeni alla storia dl concetto del tempo, trad. di R. Cristin e A. Marini, il melangolo (Opera), Genova 1991.

HEIDEGGER, M. Saggi e discorsi, trad. di G. Vattimo, Mursia (Biblioteca di filosofia), Milano 1976.

HENRY, M. Incarnazione: una filosofia della carne, trad. di G. Sansonetti, Società Editrice Internazionale (Mistero e verità), Torino 2001.

HENRY, M. «Ce que la science ne sait pas». In: La recherche 20 (mars 1989) p. 422-426.

HENRY, M. «Phénoménologie non intentionnelle: une tâche de la phénoménologie à venir». In: D. JANICAUD (ed.). L'intentionnalité en question. Entre phénoménologie et recherches cognitives, Vrin (Problèmes et controverses), Paris 1995.

HENRY, M. «Quatre principes de la phénoménologie». In: Revue de métaphysique et de morale 96 (1991) p. 3-26.

HENRY, M. «Une approche phénoménologique du christianisme». In: Études 3874 (ottobre 1997) 349-357.

HENRY, M. La barbarie, Grasset, Paris 1987.

HUSSERL, E. Glosse a Heidegger, trad. di C. Sinigaglia, Jaca Book (Lo spoglio dell'occidente), Milano 1997.

HUSSERL, E. La crisi delle scienze europee e la fenomenologia trascendentale, trad. di E. Filippini, Alberto Mondadori (Il saggiatore), Milano 1961.

HUSSERL, E. La filosofia come scienza rigorosa, trad. di C. Sinigaglia, Laterza (Universale Laterza), Roma-Bari 1994.

HUSSERL, E. Per la fenomenologia della coscienza interna del tempo, trad. di A. Marini, Franco Angeli (Filosofia), Milano 20015.

HUSSERL, E.. Prolegomeni a una logica pura. In: Ricerche logiche, trad. di G. Piana, Il saggiatore (La cultura), Milano 2015, pp. 17-197.

HUSSERL, E.; HEIDEGGER, M. Fenomenologia. Storia di un dissidio (1927), a cura di R. Cristin, Unicopli (Incroci), Napoli 1990. 
HUSSERL, E. Idee per una fenomenologia pura e per una filosofia fenomenologica, trad. di V. Costa, Einaudi [Biblioteca Einaudi], Torino 2002.

HUSSERL, E. L'idea della fenomenologia, a cura di C. Sini, Laterza (Biblioteca filosofica Laterza), Roma - Bari $2010^{6}$.

HUSSERL, E. I problemi fondamentali della fenomenologia. Lezioni sul concetto naturale di mondo, trad. di V. Costa, Quodlibet (Le forme dell'anima), Macerata 2008 (titolo della pubblicazione tedesca: Aus den Vorlessungen Grundprobleme der Phänomenologie).

MARION, J.-L. Riduzione e donazione. Ricerche su Husserl, Heidegger e la fenomenologia, trad. a cura di S. Cazzanelli, Marcianum Press (Studi filosofici), Venezia 2010.

MEESSEN, Y. Percée de l'ego. Maître Eckart en phénoménologie, Herman (De Visu), Paris 2016.

PARMENIDE. I presocratici. Testimonianze e frammenti, t. 1, trad. di P. Albertelli, Laterza [Biblioteca universale Laterza], Bari $1999^{6}$.

TOMMASO D’AQUINO. Commenti a Boezio, trad. di P. Porro, Bompiani (Testi a fronte), Milano 2007.

VIOLA, A. P. Dal corpo alla carne, Salvatore Sciascia (Facoltà teologica di Sicilia), Caltanissetta 2005. 